\title{
EL CONCEPTO DEL JUEGO Y DE LA FUSIÓN DE HORIZONTES EN LA PRODUCCIÓN DE LA NARRACIÓN METAFÓRICA
}

\author{
LUZ AURORA PIMENTEL *
}

Por medio de las siguientes reflexiones intentaré hacer, si no un replanteamiento, por lo menos una afinación importante de mi propia teoría de la narración metafórica. Para ello me serviré de la hermenéutica gadameriana como hilo conductor en esta exploración, y en especial de los conceptos de juego y de fusión de horizontes. En un primer momento describiré una parte sustancial de aquella teoría tal y como fue desarrollada en mi libro Metaphoric Narration, de 1990. Entonces planteaba yo la narración metafórica como una construcción, por medio de la lectura, de una dimensión paranarrativa que traza una línea narrativa virtual, semántica y temáticamente continua a pesar de la discontinuidad textual.

Semejante construcción paranarrativa se debe, entre otros factores, a la percepción retórica de verdaderas configuraciones narrativas o descriptivas que están programadas por el propio texto para entrar en una interacción que sigue punto por punto el proceso de metaforización tal y como lo conocemos en la figura retórica puntual, ya sea restringida a un enunciado o, de manera más extendida, en el fenómeno discursivo que se conoce como metáfora hilada o sostenida. La peculiaridad de la narración metafórica es que, sin excluir de su ámbito estas formas léxicas y discursivas, se distingue además por construir secuencias narrativas virtuales - es decir, lo que yo he llamado la dimensión paranarrativa de esta clase de relatos- que al interactuar operan de modo metafórico, sin que, necesariamente, las secuencias en sí lo sean en el nivel de la manifestación lingüística.

Hablar de narración metafórica es, entonces, hablar de efectos de sentido especiales, del orden de lo narrativo - puesto que acusan un entramado

\footnotetext{
* Facultad de Filosofia y Letras, UNAM.
} 
de transformaciones lógico-cronológicas- pero que resultan de procesos característicos de la metaforización. La narración metafórica puede darse en el nivel de la manifestación lingüistica - especialmente en la metáfora hilada - o bien en el nivel de la organización del texto. ${ }^{1}$

Acudiré, a modo de ilustración, a dos secuencias de Por el camino de Swann, de Marcel Proust, con objeto de aterrizar estos conceptos y entrar desde ahi al juego hermenéutico de su comprensión. Las secuencias elegidas constituyen complejos metafóricos de enorme densidad, tanto en su textura verbal como en el modo de articularse virtualmente en una secuencia metafórica; es decir, una tercera secuencia virtual que se produce de manera paranarrativa y genera formas de significación que no están en ninguna de las dos secuencias por separado. En la sección dedicada al mes de María, en Combray, hay un momento en el que el narrador hace una larga y hermosa descripción de las flores blancas - los famosos aubépinesespinos blancos que adornan la iglesia. Se trata de una de las descripciones verdaderamente memorables de Proust, pues la densidad de significación es tal que se irradia mucho más allá del punto en el que se inscribe para, literalmente, florecer en la segunda secuencia de los espinos blancos y rosas en Tansonville, durante los paseos por el camino de Swann, donde la experiencia se intensificará notablemente, ya que la fuerza de interpelación de estas flores desencadenará en la imaginación de Marcel una de esas experiencias de éxtasis que hemos aprendido a reconocer a partir del paradigma de la experiencia casi mistica de la magdalena sopeada en té de tila. De modo que, por la sola configuración narrativo-descriptiva, estas dos secuencias de los espinos - especialmente la segunda- entran en la declinación del paradigma de manera metafórica para significarnos una forma de acceso a la esencia de la realidad que no es necesariamente la recuperación del pasado, puesto que los espinos le dicen algo cuyo significado necesita interpretar y no desemboca necesariamente en un momento del pasado - como en el caso de la magdalena - sino en la esencia de la realidad, una realidad que es al mismo tiempo material y espiritual, encarnada en las flores casi como una proyección de la interioridad del narrador, puesto que en ellas parece encontrar la forma de su alma.

En la primera secuencia, la del mes de María, los espinos blancos descritos en la iglesia se equiparan con jovencitas engalanadas. Pero la equivalencia metafórica flores = muchachas, de muy antigua tradición, se abre a otra metáfora: las flores como abejas zumbando en un seto agreste, prepa-

${ }^{1}$ Luz Aurora Pimentel, Metaphoric Narration. Paranarrative Dimensions in 'A la recherche du temps perdu'. Toronto, University of Toronto Press, 1990, p. 35. 
rando ya la sexualización de las flores al evocar todo el proceso de polinización. Al salir de la iglesia, el narrador y su familia se encuentran con Vinteuil, el profesor de piano del pueblo, acompañado de su hija, Mlle. Vinteuil, quien más tarde habrá de convertirse en el símbolo de la otredad radical de la mujer, la terra incognita del lesbianismo que habrá de recorrer como fantasma y enigma la totalidad de En busca del tiempo perdido. Aquí, por lo pronto, la aparición de Mlle. Vinteuil, al cerrar la secuencia de los espinos blancos en la iglesia, literalmente encarna la metáfora de las muchachas en flor pero de una manera muy perturbadora, ya que la chica es identificada con las partes más excitantes de la flor, los estambres rojizos. No obstante, la excitación sexual se aborda desde el bies de la excitación visual, olfativa y gustativa:

Cuando antes de salir de la iglesia, me arrodillaba delante del altar, al levantarme sentía de pronto que se escapaba de las flores de espino un amargo y suave (dulce) olor de almendras, y advertía entonces en las flores unas manchitas rubias que, según me figuraba yo, debian de esconder ese olor, lo mismo que se oculta el sabor de un franchipán bajo la capa tostada, o el de las mejillas de la hija de Vinteuil detrás de sus pecas. ${ }^{2}$

De este modo las flores, presentadas antes como virginales por su contigüidad con el altar de la Virgen, ahora se contaminan con la presencia de Mlle Vinteuil; otras partes de las flores pasan a primer plano: por una parte el olor como de almendras que emanan, calificado paradójicamente de amargo y dulce, es comparado con el sabor de crema de almendras que Marcel imagina bajo las pecas que salpican las mejillas de Mlle. Vinteuil; por otra, los estambres -órgano masculino de la flor, y equivalencia metafórica de Mlle. Vinteuil - proyectan, no sólo su olor, sino su color rojizo a la muchacha.

Mucho más tarde, en una larguísima meditación botánico-homosexual que abre el quinto volumen, Sodoma y Gomorra, la reflexión del narrador nos llevará por el ser bisexual de las flores y la necesidad de la polinización. Si ya en botánica las flores bisexuales -es decir, aquellas dotadas de androceo y gineceo- son consideradas perfectas, Proust asume la naturaleza andrógina de las flores como una metáfora, no sólo de la homose-

2 Al final de este trabajo se ofrece, a manera de apéndice, el texto de estas dos secuencias, con sus respectivas referencias en español y francés: Marcel Proust, Por el camino de Swann. En busca del tiempo perdido. Trad. de Pedro Salinas y José Ma. Quiroga Plá, Il vols. Barcelona, Plaza \& Janés, 1975. Marcel Proust, Du côté de chez Swann. À la recherche du temps perdu, m vols. París, Gallimard, 1954. 
xualidad sino de la creación artística misma, al decir, de manera bastante críptica: "Mis reflexiones habían seguido una pendiente que describiré más tarde, y había ya deducido de la aparente astucia de las flores una consecuencia sobre toda una parte inconsciente de la obra literaria". ${ }^{3}$ La extensa narración metafórica de aquella meditación se da en la presencia de esa gran flor solitaria que es, sorprendentemente, el barón de Charlus, esperando al abejorro que vendrá a polinizarlo. Sí, en efecto, en los parajes proustianos también se oculta la sombra de los muchachos en flor.

Ahora bien, esta clara sexualización antropomórfica de las flores en la secuencia de la iglesia de Combray -que pasa por la encarnación de la metáfora en una muchacha real- se convierte en una verdadera configuración descriptiva al repetirse unas treinta páginas después en otra secuencia que, aparentemente, ya nada tiene que ver con ésta; no obstante, la configuración en esta segunda secuencia es idéntica aunque invertida. ${ }^{4}$ Por el camino que pasa por Tansonville, la propiedad de Charles Swann, el muchacho vuelve a sentirse interpelado por los espinos. De nuevo la impresión es poderosa y le deja una profunda huella, cuyo significado trata desesperadamente de interpretar. En este proceso hermenéutico, Marcel transforma una vez más a las flores en muchachas vestidas para un baile. Nuevamente aparece la metáfora de las flores como abejas, sólo que ahora sí están en un contexto campestre. Por medio de lo que se conoce como metáfora recíproca, los términos de la metáfora anterior se invierten: allá, la iglesia era el contexto diegético - es decir, no metafórico- mientras que el arbusto agreste lleno de zumbantes abejas era el foco de la metáfora; aquí, en cambio, el contexto diegético es el agreste y los términos de la arquitectura eclesiástica -ojivas, nervaduras, vitrales, altares, etcétera-se convierten en el foco de una metáfora sostenida. La significación de esta segunda secuencia - que de otro modo podría ser recibida como una mera repetición, una suerte de déjà vu-se subraya por la intensificación de los procedimientos descriptivos.

En primer lugar, ya no solamente se trata de espinos blancos, pues en una gradación cromática interesante aparecen, como en crescendo, una profusión de espinos rosas. En segundo lugar, las metáforas derivadas de la arquitectura eclesiástica se multiplican considerablemente. En la pri-

${ }^{3}$ M. Proust, Por el camino de Swann. En busca del tiempo perdido, vol. II, p. 7. En francés: "Mes réflexions avaient suivi une pente que je décrirai plus tard et j'avais déjà tiré de la ruse apparente des fleurs une conséquence sur toute une partie inconsciente de l'œuvre littéraire". (M. Proust, Du côté de chez Swann. À la recherche du temps perdu, vol. II, p. 603.)

4 Para un análisis detallado de las configuraciones descriptivas, véase el capítulo 4 de mi libro, El espacio en la ficción. México, Siglo xxı/UNAM, 2001, pp. 72-88. 
mera secuencia, las flores al final se convertían en insectos: "vibraciones semejantes a las de un seto salvaje, sembrado de vivas antenas" y "el poder irritante de insectos metamorfoseados ahora en flores"; en cambio, en la segunda secuencia, las metáforas se multiplican vertiginosamente: el seto agreste se transforma en "una serie de capillitas", los espinos se amontonan en "altarcitos", el sol proyecta "un cuadriculado de luz y sombra, como si llegara a través de un vitral"; el muchacho siente como si estuviera frente al "altar de la virgen" y "las flores así ataviadas sostenían [...] su brillante ramo de estambres, finas y radiantes molduras de estilo florido (flamboyant), como las que en la iglesia calaban la rampa del coro o los bastidores de los vitrales". En tercer lugar, vuelve a darse en esta secuencia la asociación entre las partes más excitantes de las flores y lo comestible, es decir, entre lo erótico y lo comestible: "abriendo su blanca carne de flor de fresa", "también a mí me gustaba más el queso de crema de color rosa en el que me dejaban mezclar (écraser) fresas. Y precisamente aquellas flores habían ido a escoger uno de esos tonos de cosa comestible [...]". Finalmente, la experiencia de felicidad se ha intensificado respecto de la secuencia anterior; ahora los espinos rosas le proponen un enigma semejante al de la experiencia de la magdalena: la misma profundidad de la emoción, los mismos intentos por descifrarla, por mimarla dentro de sí buscando equivalentes espirituales y estéticos para representarse esta impresión especial:

me volvía a los espinos, como se vuelve a esas obras maestras, creyendo que se les va a ver mejor después de estar un rato sin mirarlas; pero de nada servía hacerme una pantalla con las manos, para no ver otra cosa, porque el sentimiento que en mi despertaban seguia siendo oscuro e indefinido, sin poderse desprender de mi para ir a unirse a las flores.

De este modo se crea una zona de irradiación semántica de especial intensidad que nos alerta a la presencia de una significación muy particular. Tanto la repetición, modulada de esta manera, como la metáfora recíproca y sostenida, operan la articulación metafórica de las dos secuencias, porque al final de la segunda también aparece una muchacha pelirroja, casi rubia, Gilberte Swann, quien al igual que su alter ego metafórico, Mlle. Vinteuil, encarna la metáfora de las flores como muchachas; más aún, como la otra muchacha, Gilberte tiene las mismas seductoras pecas. Así, por el milagro de la metáfora, esta segunda muchacha - por su sola posición estructural en la secuencia construida con una idéntica configuración - se contamina con la sexualidad dudosa de la primera. Muchos volúmenes después se avivará repetidamente la sospecha de que Gilberte algo tuvo que ver con Mlle. Vinteuil y luego con Albertine. Empero, esta contamina- 
ción floral y sexual - este embrión de relato- surge de manera paranarrativa como una significación virtual que la articulación metafórica de las dos secuencias textualmente discontinuas propicia e irradia hacia otros complejos metafóricos posteriores. Ya no podremos leer inocentemente A la sombra de las muchachas en flor sin regresar a estas flores (y a otras aún más dudosas, como la virginidad floral y botticelliana de Mme Swann - la gran flor de esta obra - o esa otra flor altiva que es el barón de Charlus). Así, gracias a estas dimensiones paranarrativas, en lugar de tendernos cómoda y pasivamente a la sombra de estas flores, buscaremos la sombra que se cierne sobre estas muchachas en flor.

Quisiera hacer notar que esta dimensión paranarrativa generada por la articulación metafórica de dos secuencias se intensifica considerablemente debido a la apretada red de metáforas hiladas y reciprocas que las configuran, de surte que podríamos decir que estamos frente a un doble complejo metafórico: el uno en la textura verbal (la red de metáforas hiladas y recíprocas); el otro en la articulación virtual de las dos secuencias, lo cual desencadena un proceso de metaforización suplementario, una tercera secuencia virtual con una importante significación narrativa que no sería perceptible en ninguna de las dos secuencias por separado.

Ahora bien, es evidente que todos estos procesos de lectura e interpretación, tal como los he descrito, podrían ser, de manera muy productiva, creo yo, redescritos en términos de los conceptos gadamerianos del juego $\mathrm{y}$ de la fusión de horizontes. Habria que comenzar con la insistencia de Gadamer en que el juego es el modo de ser de la obra de arte..$^{5}$ Las características del juego entran en acción no sólo en la relación texto-lector, sino que, al interior del propio texto, en el caso de la narración metafórica, es posible encontrar esos mismos rasgos del juego, desdoblados y como en espejo, en la interacción de ciertas secuencias que se articulan virtualmente en un modo metafórico. Quisiera destacar sólo algunos aspectos del juego -y no olvidemos que, como dice Gadamer, "todo destacar algo vuelve simultáneamente visible aquello de lo que se destaca". 6

En primer lugar, me detendré en el concepto del juego como proceso medial. Si el modo de ser del juego es la autorrepresentación, sólo accede a ella por medio de los jugadores; ${ }^{7}$ es decir, que se trata siempre de una "representación para un espectador". 8 Si bien Gadamer toma en un primer

${ }^{5}$ Hans-Georg Gadamer, Verdad y método: fundamentos de una hermenéutica filosófica. Salamanca, Sígueme, 1991, p. 143.

6 Ibid., p. 376.

7 Cf. ibid., p. 145.

8 Ibid., p. 153. (Cursivas en el original.) 
momento la obra dramática y la musical como ilustración del ser de la obra de arte como representación, esto es por lo evidente de la mediación, pues "sólo hay verdadero drama cuando se lo representa y, desde luego, la música tiene que sonar.. ${ }^{9}$ Pero esto es extensible a la obra de arte verbal, pues si materialmente el texto puede existir de manera autónoma, no hay obra y, por ende, significación, sin su lectura. Texto y lector se convierten así en los jugadores que construyen un espacio lúdico definido por las reglas del juego. Ahora bien, en el caso de la narración metafórica -actividad plenamente lúdica, si hay alguna- el espacio del juego está constituido por las reglas mismas de lo que podriamos llamar la percepción retórica, una competencia requerida del lector sin la cual no se activaría la articulación metafórica de las secuencias. Por una parte, el texto se organiza de tal manera que programa el inicio del juego metafórico para que el lector construya la secuencia paranarrativa: en nuestro ejemplo, la metáfora de base flores = muchachas, aunada a la metáfora reciproca de las flores en contextos eclesiástico y agreste $\mathrm{y}$, finalmente, la configuración narrativo-descriptiva de la muchacha al final de cada secuencia, todo lo cual provoca una intensa actividad interpretativa, no sólo en el lector, sino, especularmente, en el mismo narrador, quien trata sin éxito de descifrar el significado espiritual de la impresión combinada que le causan las flores que él transfigura en muchachas y las muchachas a las que su imaginación luego revierte en flores. Lo interesante, además, es que estas reglas conforman el horizonte tanto del texto como del lector y tienen su punto de convergencia en la tradición.

El horizonte es [...] algo en lo que hacemos nuestro camino y que hace el camino con nosotros. El horizonte se desplaza al paso de quien se mueve. También el horizonte del pasado, del que vive toda vida humana y que está ahí bajo la forma de la tradición, se encuentra en perpetuo movimiento [...] Cuando nuestra conciencia histórica se desplaza hacia horizontes históricos esto no quiere decir que se traslade a mundos extraños, a los que nada vincula con el nuestro; por el contrario todos ellos juntos forman ese gran horizonte que se mueve por sí mismo y que rodea la profundidad histórica de nuestra autoconciencia más allá de las fronteras del presente. En realidad es un único horizonte el que rodea cuanto contiene en sí misma la conciencia histórica. El pasado propio y extraño al que se vuelve la conciencia histórica forma parte del horizonte móvil desde el que vive la vida humana y que determina a ésta como su origen y su tradición. ${ }^{10}$

9 Ibid., p. 161.

10 Ibid., p. 375. 
En el ejemplo que he venido utilizando, basta con aislar dos reglas que hacen horizonte: por una parte, la competencia retórica $\mathrm{y}$, por otra, la muy trillada metáfora reversible flores $=$ muchachas $=$ flores. Se trata de un horizonte que el lector puede compartir por estar inmerso en esa tradición; no obstante, al mismo tiempo que le es familiar, también le es extraño, pues el juego de la lectura lo obliga a responder a iniciativas nuevas provenientes del texto; lo obliga, entre otras cosas, a extender sus nociones de lo que es una metáfora para leerla de otro modo, no sólo narrativa sino estereoscópicamente, por así decirlo. Lo incita, asimismo, a des-banalizar la metáfora de las flores como muchachas, a mirarla con otros ojos, a renovarla, en pocas palabras, y no como alguien ajeno a esa metáfora-idea, sino como alguien inmerso en esa tradición para quien flores y muchachas se han asociado innumerables veces. Más aún, ese proceso de des-banalización se da en una fusión que integra el horizonte proustiano, el cual, a su vez, ha incorporado la metáfora trillada al horizonte de la botánica para activar la dimensión andrógina de las flores $\mathrm{y}$, por extensión, de sus muchachas en flor; extensión que más tarde habrá de incorporar también a la homosexualidad al convertir a un hombre en flor - las yuxtaposiciones y las paradojas no podrían ser más sorpresivas.

Así, el nuevo significado es lo ajeno de lo cual se apropia el lector, pues, como dice Gadamer

revelar lo que es extraño no significa simplemente la reconstrucción histórica del mundo en el que la obra tenía su significado y función originales. Significa también aprehender lo que se nos dice que es siempre más que el significado declarado y comprendido [...] hay una sorpresa en el significado de lo dicho [...] El elemento de sorpresa se basa en esto. "Tan cierto, tan siendo" [So wahr, so seiend], es algo que no se conoce de otra manera."11

Habria que subrayar que la sola forma verbal - seiend- apunta claramente a esta plenitud del ser como proceso, como algo que está siempre siendo. Me parece además que, aunque no es el propósito ostensible de Gadamer en estas reflexiones, nada describe mejor lo que se quiere decir con los términos significación narrativa: un modo dinámico, temporal, de aprehender el significado de una experiencia humana que no sería posible de otra manera porque la narrativa, como la identidad misma, es siempre siendo, "pues - como lo apunta Proust- uno no se realiza más que de manera sucesiva" (car on ne se réalise que successivement).

11 H.-G. Gadamer, "Aesthetics and Hermeneutics", en Philosophical Hermeneutics. Berkeley, University of California Press, 1976, p. 101. 
Ahora bien, la aprehensión de este nuevo significado es una forma de integración, de fusión de horizontes - una mirada estereoscópica, como la llamaria Ricoeur - y finalmente un enriquecimiento de la propia experiencia, una ampliación de horizonte, porque, como bien lo dice Gadamer, "ganar un horizonte quiere decir siempre aprender a ver más allá de lo cercano y de lo muy cercano, no desatenderlo, sino precisamente verlo mejor, integrándolo en un todo más grande y en patrones más correctos".12 La integración se da en esa fusión que permite esta ampliación del horizonte, de mundo, pues "todo lo que está dado como ente, está dado como mundo y lleva consigo el horizonte del mundo".13

Algo en lo que quisiera yo insistir, finalmente, es la posibilidad de ver en la narración metafórica una estructura en abismo de la situación hermenéutica que rige la recepción de la obra de arte como un todo. Debido al carácter eminentemente temporal de la lectura, la primera secuencia se convierte para el lector en el horizonte sobre el que se destaca la segunda; es el pasado que se presentifica en el momento de leer la segunda secuencia. Más aún, semejante lectura estereoscópica se da en un juego de vaivén en el que se van marcando los patrones semánticos, las repeticiones que abren el espacio de la figura narrativa que no es otro que el del juego mismo. Este ir y venir también implica un desplazamiento y una integración $\mathrm{y}$, por lo tanto, un ascenso hacia una generalidad superior que rebasa tanto la particularidad de una secuencia como la de la otra con la que se articula, para darnos una tercera dimensión de significado que se propone como la esencia de la significación narrativa de estas secuencias, ${ }^{14}$ esencia a la que se llega por exceso, pues estas formas de significación narrativa no se observan en ninguna de las dos secuencias, sino que son el producto de la interacción metafórica virtual: "el lenguaje del arte es un exceso de significado presente en la propia obra. El carácter de inagotable que distingue al lenguaje del arte de toda traducción a conceptos descansa en este excedente de sentido". 15 De este modo, si el arte se distingue por una especie de surplus de significación, esto no es sino el resultado de la búsqueda de un equivalente artístico de la experiencia profunda de la realidad, de la naturaleza radical e irreductiblemente individual de toda experiencia que se traduce en un mundo. Dejémosle a Proust la última palabra.

\footnotetext{
12 H.-G. Gadamer, Verdad y método: fundamentos de una hermenéutica filosófica, p. 375. 13 Ibid., p. 309.

14 Cf. ibid., p. 375.

15 H.-G. Gadamer, "Aesthetics and Hermeneutics", en op. cit., p. 102.
} 
La grandeza del arte verdadero [...] consistía en recobrar, en recoger, en hacernos conocer esta realidad lejos de la cual vivimos, de la que nos apartamos más y más, a medida que gana en espesor y en impermeabilidad el conocimiento convencional que le sustituimos, y correriamos el riesgo de morir sin haber conocido esa realidad, que es sencillamente nuestra vida.

La verdadera vida, la vida por fin descubierta e iluminada, la única vida en consecuencia realmente vivida, es la literatura. Esa vida que en un sentido habita a cada instante lo mismo en los hombres que en el artista. Pero no la ven porque no tratan de iluminarla. $Y$ asi su pasado se recarga de innumerables clichés que permanecen inútiles porque la inteligencia no los ha revelado. Recoger nuestra vida y también la vida de los demás, pues el estilo, para el escritor lo mismo que para el pintor, es una cuestión de visión y no de técnica, es la revelación que resultaría imposible, por los medios directos y conscientes, de la diferencia cualitativa que hay en la manera bajo la que se nos aparece el mundo, diferencia que, si no existiese el arte, continuaría siendo el eterno secreto de cada uno. Solamente por medio del arte podemos salir de nosotros, saber lo que otro ve de este universo que no es el mismo que el nuestro y cuyos paisajes habrian continuado siéndonos tan desconocidos como los que pueden existir en la luna. Gracias al arte, en lugar de ver un solo mundo, el nuestro, lo vemos multiplicarse, y tantos como artistas originales, tenemos otros tantos mundos a nuestra disposición. ${ }^{16}$

${ }^{16}$ M. Proust, Por el camino de Swann. En busca del tiempo perdido, vol. II, pp. 1453-1455. En francés: "La grandeur de l'art véritable... c'était de retrouver, de ressaisir, de nous faire connaitre cette réalité loin de laquelle nous vivons, de laquelle nous nous écartons de plus en plus au fur et à mesure que prend plus d'épaisseur et d'imperméabilité la connaissance conventionnelle que nous lui substituons, cette réalité que nous risquerions fort de mourir sans avoir connue, et qui est tout simplement notre vie.

La vraie vie, la vie enfin découverte et éclaircie, la seule vie par conséquent pleinement vécue, c'est la littérature. Cette vie qui, en un sens, habite à chaque instant chez tous les hommes aussi bien que chez l'artiste. Mais ils ne la voient pas, parce qu'ils ne cherchent pas à l'éclaircir. Et ainsi leur passé est encombré d'innombrables clichés qui restent inutiles parce que l'intelligence ne les a pas "développés". Notre vie; et aussi la vie des autres; car le style pour l'écrivain aussi bien que la couleur pour le peintre est une question non de technique mais de vision. Il est la révélation, qui serait impossible par des moyens directs et conscients, de la différence qualitative qu'il y a dans la façon dont nous apparait le monde, différence qui, s'il n'y avait pas l'art, resterait le secret éternel de chacun. Par l'art seulement nous pouvons sortir de nous, savoir ce que voit un autre de cet univers qui n'est pas le même que le nôtre et dont les paysages nous seraient restés aussi inconnus que ceux qu'il peut y avoir dans la lune. Grâce à l'art, au lieu de voir un seul monde, le nôtre, nous le voyons se multiplier, et autant qu'il y a d'artistes originaux, autant nous avons de mondes à notre disposition... Pléiade" (M. Proust, Du côté de chez Swann. À la recherche du temps perdu, vol. uI, pp. 895-896.) 


\section{APÉNDICE}

Recuerdo que fue en el mes de María cuando empecé a tomar cariño a las flores de espino. En la iglesia, tan santa, pero donde teníamos derecho a entrar, no sólo estaban posadas en los altares, inseparables de los misterios en cuya celebración participaban, sino que dejaban correr entre las luces y los floreros santos sus ramas atadas horizontalmente unas a otras en aparato de fiesta, y embellecidas aún más por los festones de las hojas, entre las que lucían, profusamente sembrados, como en la cola de un traje de novia, los ramitos de capullos blanquísimos [...] Más arriba abríanse las corolas, aquí y allá, con desafectada gracia, reteniendo con negligencia suma, como último y vaporoso adorno, el ramito de estambres, tan finos como hilos de la Virgen, y que les prestaban una suave veladura; y cuando yo quería seguir e imitar en lo hondo de mi ser el movimiento de su floración, lo imaginaba como el cabeceo rápido y voluble de una muchacha blanca, distraida y vivaz, con mirar de coquetería y pupilas disminuidas. M. Vinteuil venía a sentarse con su hija a nuestro lado [...] Cuando [M1le. Vinteuil] acababa de pronunciar una palabra, oíala con la mente de la persona a quien iba dirigida, se alarmaba por las malas interpretaciones que pudieran dársele, y bajo la figura hombruna de aquel diablo, se alumbraba y se recortaban, como por transparencia, los finos rasgos de una muchacha llorosa.

Cuando antes de salir de la iglesia, me arrodillaba delante del altar, al levantarme sentía de pronto que se escapaba de las flores de espino un amargo y suave [dulce] olor de almendras, y advertía entonces en las flores unas manchitas rubias que, según me figuraba yo, debian de esconder ese olor, lo mismo que se oculta el sabor de un franchipán bajo la capa tostada, o el de las mejillas de la hija de Vinteuil detrás de sus pecas. A pesar de la callada quietud de las flores de espino, ese olor intermitente era como un murmullo de intensa vida, la cual prestaba al altar vibraciones semejantes a las de un seto salvaje, sembrado de vivas antenas, cuya imagen nos la traían al pensamiento algunos estambres casi rojos que parecían conservar aún la virulencia primaveral y el poder irritante de insectos metamorfoseados ahora en flores. ${ }^{17}$

[C'est au mois de Marie que je me souviens d'avoir commencé à aimer les aubépines. N'étant pas seulement dans l'église, si sainte, mais où nous avions le droit d'entrer, posées sur l'autel même, inséparable des mystères

17 M. Proust, Por el camino de Swann. En busca del tiempo perdido, vol. I, pp. 112-114. 
à la célébration desquels elles prenaient part, elles faisaient courir au milieu des flambeaux et des vases sacrés leurs branches attachées horizontalement les unes aux autres en un apprêt de fête et qu'enjolivaient encore les festons de leur feuillage sur lequel étaient semés à profusion, comme sur une traîne de mariée, de petits bouquets de boutons d'une blancheur éclatante. Mais sans oser regarder qu'à la dérobée, je sentais que ces apprêts pompeux étaient vivants et que c'était la nature elle-même qui, en creusant ces découpures dans les feuilles, en ajoutant l'ornement suprême de ces blancs boutons, avait rendu cette décoration digne de ce qui était à la fois une réjouissance populaire et une solennité mystique. Plus haut s'ouvraient leurs corolles çà et là avec une grâce insouciante, retenant si négligemment, comme un dernier et vaporeux atour, le bouquet d'étamines, fines comme des fils de la Vierge, qui les embrumait tout entières, qu'en suivant, qu'en essayant de mimer au fond de moi le geste de leur efflorescence, je l'imaginais comme si ç'avait été le mouvement de tête étourdi et rapide, au regard coquet, aux pupilles diminuées, d'une blanche jeune fille, distraite et vive. M. Vinteuil était venu avec sa fille se placer à côte de nous... Quand elle venait de prononcer une parole, elle l'entendait avec l'esprit de ceux à qui elle l'avait dite, s'alarmait des malentendus possibles, et on voyait s'éclairer, se découper comme par transparence, sous la figure homasse du «bon diable», les traits plus fins d'une jeune fille éplorée.

Quand, au moment de quitter l'église, je m'agenouillai devant l'autel, je sentis tout d'un coup, en me relevant, s'échapper des aubépines une odeur amère et douce d'amandes, et je remarquai alors sur les fleurs de petites places plus blondes sous lesquelles je me figurai que devait être cachée cette odeur, comme, sous les parties gratinées, le goût d'une frangipane ou, sous leurs taches de rousseur, celui des joues de Mlle. Vinteuil. Malgré la silencieuse immobilité des aubépines, cette intermittente odeur était comme le murmure de leur vie intense dont l'autel vibrait ainsi qu'une haie agreste visitée par de vivantes antennes, auxquelles on pensait en voyant certaines étamines presque rousses qui semblaient avoir gardé la virulence printanière, le pouvoir irritant, d'insectes aujourd'hui métamorphosés en fleurs.] ${ }^{18}$

En el caminito susurraba [bourdonnant] el aroma de los espinos blancos. El seto formaba como una serie de capillitas, casi cubiertas por montones de flores que se agrupaban, formando a modo de altarcitos de mayo; y

18 M. Proust, Du còté de chez Swann. À la recherche du temps perdu, vol. I, pp. 112-114. 
abajo el sol extendía por el suelo, un cuadriculado de luz y sombra, como si llegara a través de un vitral; el olor difundíase tan untuosamente, tan delimitado en su forma, como si me encontrara delante del altar de la Virgen, y las flores así ataviadas sostenían, con distraído ademán, su brillante ramo de estambres, finas y radiantes molduras de estilo florido, como las que en la iglesia calaban la rampa del coro o los bastidores de los vitrales, abriendo su blanca carne de flor de fresa [...]

Pero de nada me servía quedarme parado delante de los espinos, respirando su olor invisible y fijo, presentándosele a mi pensamiento, que no sabía lo que hacer con él, perdiéndolo y volviendo a encontrarlo, entregándome al ritmo que lanzaban sus flores, ya a un lado, ya a otro, con gozo juvenil e intervalos inesperados, como algunos intervalos musicales: ofrecíanme indefinidamente la misma seducción, con profusión inagotable; pero sin dejarme ahondar más adentro, como esas melodias que se cantan y se cantan sin penetrar nunca su secreto. Íbame de su lado un momento para tornar a ellas con fuerzas frescas $[. .$.

Luego me volvía a los espinos, como se vuelve a esas obras maestras, creyendo que se les va a ver mejor después de estar un rato sin mirarlas; pero de nada servía hacerme una pantalla con las manos, para no ver otra cosa, porque el sentimiento que en mi despertaban seguía siendo oscuro e indefinido, sin poderse desprender de mí para ir a unirse a las flores. Las cuales no me ayudaban a aclarar mi sentimiento, sin que yo pudiera pedir a otras flores que los satisficieran. Entonces, entregándome a esa alegría que se siente al ver una obra de nuestro pintor favorito que difiere de las que conocemos [...] mi abuelo me llamaba, y señalándome el seto de Tansonville, me decía: "Mira tú, que tanto te gustan los espinos; mira ese espino rosa qué bonito es". Y, en efecto, era un espino, pero éste de color rosa y aún más hermoso que los blancos. También estaba vestido de fiesta - religiosa, las únicas festividades verdaderas, porque no hay un capricho contingente que las aplique como las fiestas mundanas a un día cualquiera, que no esté especialmente consagrado a ellas, y que nada tiene de esencialmente festivo-, pero más ricamente vestido, porque las flores [estaban] pegadas a la rama, unas encima de otras, sin dejar ningún hueco sin decorar, como los pompones que adornan los cayados de estilo rococó, eran de color.

[...] También a mí me gustaba más el queso de crema de color rosa en el que me dejaban mezclar (écraser) fresas. Y precisamente aquellas flores habian ido a escoger uno de esos tonos de cosa comestible, o de tierno realce de un traje para fiesta mayor $[\ldots]$

En lo alto de las ramas [...] pululaban mil capullitos de tono más pálido, que, entreabriéndose, dejaban ver, como en el fondo de una copa de már- 
mol rosa, ágatas sangrientas, y delataban aún más claramente que las flores, la esencia particular e irresistible del espino, que donde quiera que eche brote o florezca, no sabía hacerlo más que con color de rosa. Intercalado en el seto pero diferenciándose de él, como una jovencita en traje de fiesta, entre personas desaseadas que se quedarán en casa, ya preparado para el mes de María, del que parecía estar participando, brillaba sonriente, con su fresco vestido rosa, el arbusto católico y delicioso [...]

[...] De repente me paré, sin poder moverme, como sucede cuando vemos algo que no sólo va dirigido a nuestro mirar, sino que requiere más profundas percepciones y se adueña de nuestro ser entero. Una chica de un rubio rojizo que, al parecer, volvía de paseo, y que llevaba en la mano una azada de jardín, nos miraba, alzando el rostro, salpicado de manchitas de color rosa $[\ldots]^{19}$

[Je le trouvai tout bourdonnant de l'odeur des aubépines. La haie formait comme une suite de chapelles qui disparaissaient sous la jonchée de leurs fleurs amoncelées en reposoir; au-dessous d'elles, le soleil posait à terre un quadrillage de clarté, comme s'il venait de traverser une verrière; leur parfum s'étendait aussi onctueux, aussi délimité en sa forme que si j'eusse été devant l'autel de la Vierge, et les fleurs, aussi parées, tenaient chacune d'un air distrait son étincelant bouquet d'étamines, fines et rayonnantes nervures de style flamboyant comme celles qui à l'église ajouraient la rampe du jubé ou les meneaux du vitrail et qui s'épanouissaient en blanche chair de fleur de fraisier [...]

Mais j'avais beau rester devant les aubépines à respirer, à porter devant ma pensée qui ne savait ce qu'elle devait en faire, à perdre, à retrouver leur invisible et fixe odeur, à m'unir au rythme qui jetait leurs fleurs... elles m'offraient indéfiniment le même charme avec une profusion inépuisable, mais sans me laisser approfondir davantage, comme ces mélodies qu'on rejoue cent fois de suite sans descendre plus avant dans leur secret. Je me détournais d'elles un moment, pour les aborder ensuite avec des forces plus fraîches $[\ldots]$

Puis je revenais devant les aubépines comme devant ces chefs-d'œuvre dont on croit qu'on saura mieux les voir quand on a cessé un moment de les regarder, mais j'avais beau me faire un écran de mes mains pour n'avoir qu'elles sous les yeux, le sentiment qu'elles éveillaient en moi restait obscur et vague, cherchant en vain à se dégager, à venir adhérer à leurs fleurs. Elles ne m'aidaient pas à l'éclaircir, et je ne pouvais demander à

${ }^{19}$ M. Proust, Por el camino de Swann. En busca del tiempo perdido, vol. I, pp. 137-140. 
d'autres fleurs de le satisfaire. Alors, me donnant cette joie que nous éprouvons quand nous voyons de notre peintre préféré une oeuvre qui diffère de celles que nous connaissions [...] mon grand-père, m'appelant et me désignant la haie de Tansonville, me dit: "Toi qui aimes les aubépines, regarde un peu cette épine rose, plus belle encore que les blanches. Elle aussi avait une parure de fête, - de ces seules vraies fêtes que sont les fêtes religieuses, puisqu'un caprice contingent ne les applique pas comme les fêtes mondaines à un jour quelconque qui ne leur est pas spécialement destiné, qui n'a rien d'essentiellement férié- mais une parure plus riche encore, car les fleurs attachées sur la branche, les unes au-dessus des autres, de manière à ne laisser aucune place qui ne fût décorée, comme des pompons qui engurilandent une houlette rococo, étaient "en couleur» [...] Moimême j'appréciais plus le fromage à la crème rose, celui où l'on m'avait permis d'écraser des fraises. Et justement ces fleurs avaient choisi une de ces teintes de chose mangeable ou de tendre embellissement à une toilette pour une grande fête $[\ldots]$

Au haut des branches [...] pullulaient mille petits boutons d'une teinte plus pâle qui, en s'entr'ouvrant, laissaient voir, comme au fond d'une coupe de marbre rose, de rouges sanguines, et trahissaient, plus encore que les fleurs, l'essence particulière, irrésistible de l'épine, qui, partout où elle bourgeonnait, où elle allait fleurir, ne le pouvait qu'en rose. Intercalé dans ,milieu de personnes en négligé qui resteront à la maison, tout prêt pour le mois de Marie, dont il semblait faire partie déjà, tel brillait en souriant dans sa fraîche toilette rose l'arbuste catholique et délicieux [...]

[...] Tout à coup, je m'arrêtai, je ne pus plus bouger, comme il arrive quand une vision ne s'adresse pas seulement à nos regards, mais requiert des perceptions plus profondes et dispose de notre être tout entier. Une fillette d'un blond roux, qui avait l'air de rentrer de promenade et tenait à la main une bêche de jardinage nous regardait, levant son visage semé de taches roses. $]^{20}$

${ }^{20}$ M. Proust, Du côté de chez Swann. À la recherche du temps perdu., vol. I, pp. 138-140. 